\title{
Acute Inferior Myocardial Infarction Presenting as Anterior ST Segment Elevation Myocardial Infarction on ECG
}

\author{
Muhammad Ali ${ }^{*}$, Angelos G. Rigopoulos ${ }^{1}$, \\ Jan Lukas Prüser', Marios Matiakis' ${ }^{1}$, Khaldoun Ali², \\ Alexander Vogt ${ }^{1}$, Michel Noutsias ${ }^{1}$
}

${ }^{1}$ Mid-German Heart Center, Department of Internal Medicine III, Division of Cardiology, Angiology and Intensive Medical Care, University Hospital Halle, Martin-Luther-University Halle-Wittenberg, Halle, Germany

${ }^{2}$ Department of Thoracic and Cardiovascular Surgery, Klinikum Braunschweig, Germany

Email: *muhammad.ali@uk-halle.de, angelos.rigopoulos@uk-halle.de, jan.prueser@uk-halle.de, marios.matiakis@uk-halle.de,

khaldounali4@hotmail.com, alexander.vogt@uk-halle.de,michel.noutsias@uk-halle.de

How to cite this paper: Ali, M., Rigopoulos, A.G., Prüser, J.L., Matiakis, M., Ali, K., Vogt, A. and Noutsias, M. (2018) Acute Inferior Myocardial Infarction Presenting as Anterior ST Segment Elevation Myocardial Infarction on ECG. World Journal of Cardiovascular Diseases, 8, 518-522.

https://doi.org/10.4236/wjcd.2018.811050

Received: October 25, 2018

Accepted: November 23, 2018

Published: November 26, 2018

Copyright $\odot 2018$ by authors and Scientific Research Publishing Inc. This work is licensed under the Creative Commons Attribution International License (CC BY 4.0).

http://creativecommons.org/licenses/by/4.0/

\begin{abstract}
A 51-year-old man presented to the emergency department with acute substernal chest pain. ECG showed ST-segment elevation in the left precordial leads. Coronary angiography demonstrated an occlusion of the right coronary artery (RCA) and no significant stenosis in left anterior descending coronary artery (LAD). The occlusion of a non-dominant RCA may result in anterior ST-segment elevation ECG changes, which could disorient both general and interventional cardiologists.
\end{abstract}

\section{Keywords}

Myocardial Infarction, Occlusion, Electrocardiogram, Coronary Angiography

\section{Introduction}

The ST-segment elevation in the precordial leads $V_{1}-V_{6}$ is characteristically a hallmark of ST-segment elevation myocardial infarction (STEMI) of the anterior left myocardium, which is caused by an occlusion of the left anterior descending (LAD) coronary artery [1] [2]. Here, we report a case of a patient with acute anterior myocardial infarction who underwent acute coronary angiography. In contrast to the expected LAD occlusion, we found an occlusion of the right coronary artery (RCA), which was treated by primary percutaneous coronary intervention (pPCI). 


\section{Case Report}

A 51-year-old man presented to the emergency department with substernal chest pain of 40 minutes duration. The chest pain was associated with nausea and sweating. He had a medical history of hypertension. On admission, his blood pressure was $170 / 85 \mathrm{mmHg}$, heart rate 54 beats per minute, and respiratory rate of 18 breaths per minute. An electrocardiogram (ECG) showed ST-segment elevation in the left precordial leads $\mathrm{V}_{2}$ through $\mathrm{V}_{4}$, T-wave inversion in leads II, III, and aVF (Figure 1). The troponin I level was $92 \mathrm{ng} / \mathrm{L}$ (normal value $<14$ $\mathrm{ng} / \mathrm{L}$ ). The patient underwent acute coronary angiography, which demonstrated no significant stenosis in the LAD (Figure 2(a)). The dominant left circumflex coronary artery (LCX) was mildly diseased (Figure 2(b)). In contrast to the ST-segment elevations in the left precordial leads $\mathrm{V}_{2}$ through $\mathrm{V}_{4}$, a complete occlusion of the proximal RCA (Figure 2(c)) was documented. The RCA occlusion was treated successfully by primary percutaneous coronary intervention (pPCI), including the implantation of a drug-eluting stent (Figure 2(d)). After the pPCI, the patient was free of angina pectoris and hemodynamically stable. The troponin I level rose to a peak of $860 \mathrm{ng} / \mathrm{L}$ (normal value $<14 \mathrm{ng} / \mathrm{L}$ ) 11 hours after the procedure. The abnormal ST-segment elevations remained unchanged in the follow-up post-procedural ECGs. The patient was set on guideline-directed medical therapy, and was referred to a cardiac rehabilitation clinic.

\section{Discussion}

The ECG stays the essential diagnostic method of coronary artery disease. On 12-lead ECG, the ST-segment elevation in the precordial leads usually indicates a

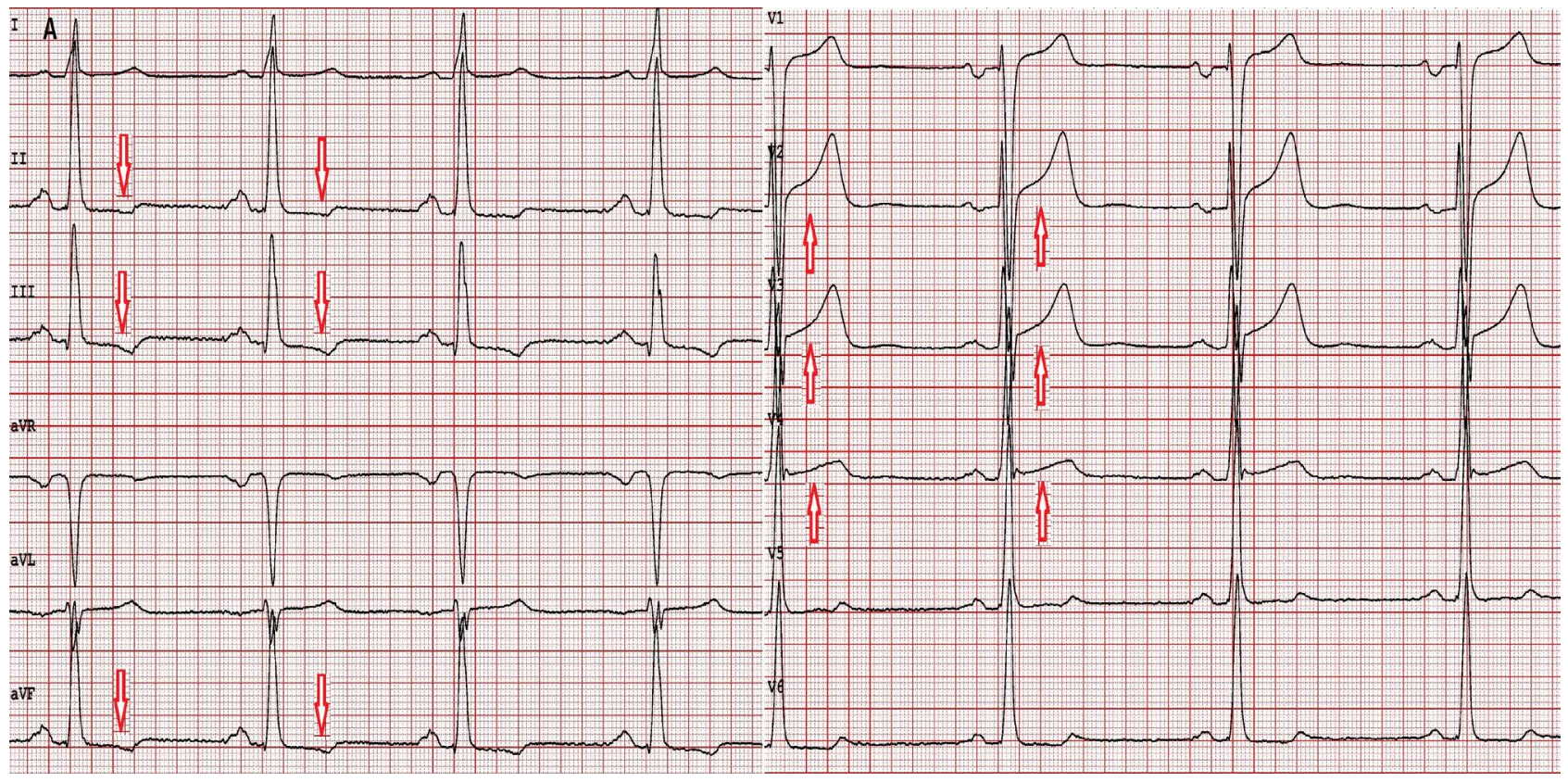

Figure 1. A 12 lead electrocardiogram at patient admission, showing ST-segment elevations in the left precordial leads V2 through V4 (arrows $\uparrow$ ), T-wave inversion in leads II, III and aVF (arrows $\downarrow$ ). 


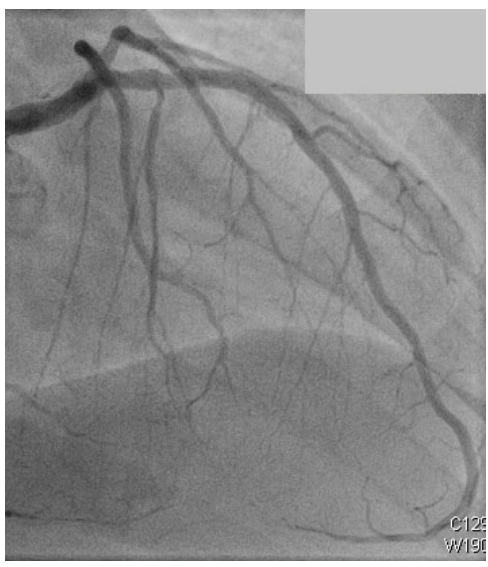

(a)

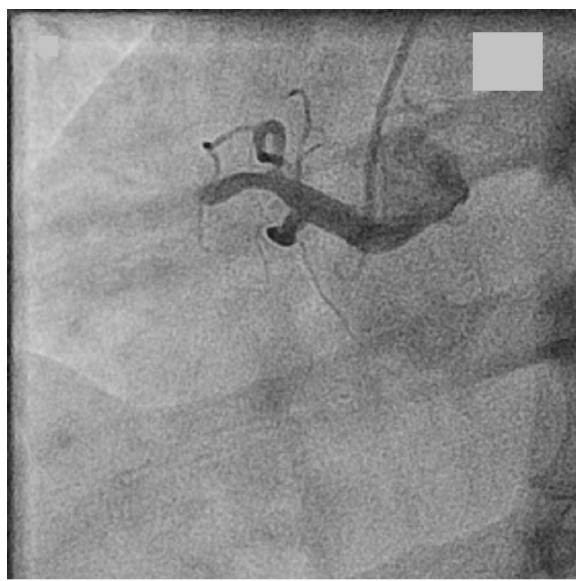

(c)

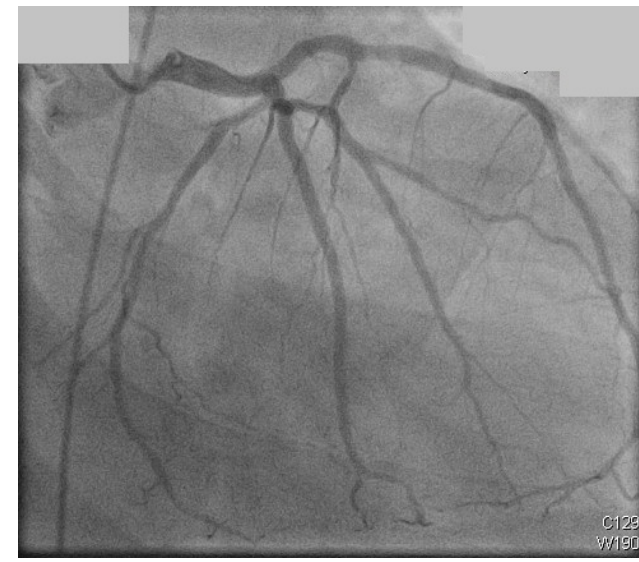

(b)

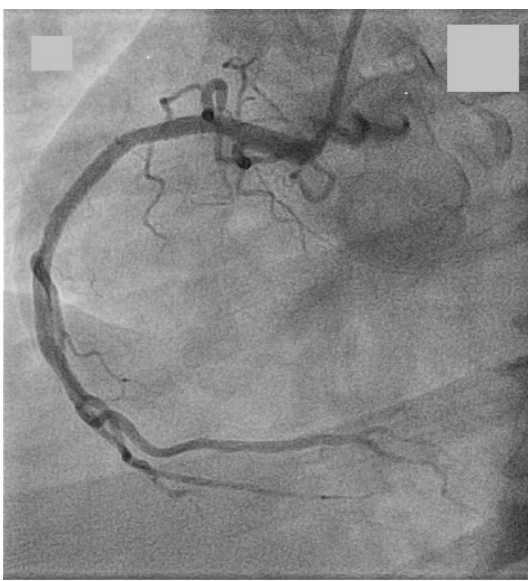

(d)

Figure 2. (a) Coronary angiography shows the LAD without significant stenosis. (b) Coronary angiography shows a dominant LCX without significant stenosis. (c) Coronary angiography shows a complete occlusion of the proximal RCA. (d) Coronary angiography of the RCA post recanalization and pPCI.

STEMI of the anterior myocardial wall due to an acute occlusion of the LAD or one of its diagonal branches [1] [2].

In case of a dominant-RCA occlusion, it is presupposed that the electrical changes of inferior left ventricular infarction mask the anterior changes of right ventricular infarction, resulting in a typical pattern of inferior ST-segment elevation myocardial infarction. A non-dominant RCA supplies only the right ventricular free wall, and the occlusion of this artery is not associated with inferior left ventricular infarction, but may result in anterior ST-segment elevation ECG changes [3].

Several reports described the association between anterior ST-segment elevation and the occlusion of a dominant RCA in the context of a collateralization from branches of the left coronary artery system [3] [4] [5] [6] [7]. However, there are only scarce data describing the relationship between anterior ST-segment elevation and the occlusion of a non-dominant RCA [8] [9] [10] [11]. Fin et al. revealed a right ventricular myocardial infarction caused by a 
critically stenotic non-dominant RCA in a patient with anterior ST-segment elevation using magnetic resonance imaging [12]. In this case report we described a rare case, in which the occlusion of RCA caused atypically anterior ST-segment elevation on ECG.

We believe that this case will be of interest to medical students, general practitioners, internists, general and interventional cardiologists. We recommend that interventional cardiologists should be aware and prepared for such cases; especially in the selection of an appropriate PCI strategy.

\section{Conclusion}

The anterior ST-segment elevation in precordial ECG leads could disorient the interventional cardiologists since the occlusion of a non-dominant RCA may be responsible for this paradoxical phenomenon.

\section{Disclosures}

Verbal and written informed consent was obtained from the patient for his de-identified information to be published in this article.

\section{Funding Support}

No agency or funding from any source was received for this work.

\section{Conflicts of Interest}

$\mathrm{AM}, \mathrm{AR}$ and $\mathrm{MN}$ received honoraria for presentations from AstraZeneca.

\section{References}

[1] Zimetbaum, P.J. and Josephson, M.E. (2003) Use of the Electrocardiogram in Acute Myocardial Infarction. New England Journal of Medicine, 348, 933-940. https://doi.org/10.1056/NEJMra022700

[2] Ali, M., Lange, S.A., Wittlinger, T., Lehnert, G., Behrend, S., Ziadeh, B., Ali, K., Sakellaropoulos, S., Ganchev, G., Rigopoulos, A.G. and Noutsias, M. (2018) Direct Transfer of STEMI Patients to Cardiac Catheterization Laboratory: Prognostic Relevance for In-Hospital Mortality. Herz. [Epub Ahead of Print] https://doi.org/10.1007/s00059-017-4673-Z

[3] van der Bolt, C.L., Vermeersch, P.H. and Plokker, H.W. (1996) Isolated Acute Occlusion of a Large Right Ventricular Branch of the Right Coronary Artery Following Coronary Balloon Angioplasty. The Only True 'Model' to Study ECG Changes in Acute, Isolated Right Ventricular Infarction. European Heart Journal, 17, 247-250. https://doi.org/10.1093/oxfordjournals.eurheartj.a014841

[4] Muhammad, K.I. and Kapadia, S.R. (2008) Anterior ST-Segment Elevation with Right Coronary Artery Occlusion: A Unique Case of Isolated Right Ventricular Infarction. Angiology, 59, 622-624. https://doi.org/10.1177/0003319707305464

[5] Wilson, J.M., Kalife, G., Rogers, M., Strickman, N.E. and Massumi, A. (1996) Unusual Electrocardiographic Presentation of Right Ventricular Myocardial Infarction. Texas Heart Institute Journal, 23, 305-309.

[6] Koh, T.W., Coghlan, J.G. and Lipkin, D.P. (1996) Anterior ST Segment Elevation 
Due to Isolated Right Ventricular Infarction during Right Coronary Angioplasty. International Journal of Cardiology, 54, 201-206. https://doi.org/10.1016/0167-5273(96)02610-1

[7] Acikel, M., Yilmaz, M., Bozkurt, E., Gurlertop, Y. and Kose, N. (2003) ST Segment Elevation in Leads V1 to V3 Due to Isolated Right Ventricular Branch Occlusion during Primary Right Coronary Angioplasty. Catheterization and Cardiovascular Interventions, 60, 32-35. https://doi.org/10.1002/ccd.10610

[8] Eichhofer, J. and Curzen, N. (2005) Images in Cardiovascular Medicine. Unexpected Profound Transient Anterior ST Elevation after Occlusion of the Conus Branch of the Right Coronary Artery during Angioplasty. Circulation, 111, e113-e114. https://doi.org/10.1161/01.CIR.0000157152.65623.23

[9] Porter, A., Herz, I. and Strasberg, B. (1997) Isolated Right Ventricular Infarction Presenting as Anterior Wall Myocardial Infarction on Electrocardiography. Clinical Cardiology, 20, 971-973. https://doi.org/10.1002/clc.4960201115

[10] Celik, T., Yuksel, U.C., Kursaklioglu, H., Iyisoy, A., Kose, S. and Isik, E. (2006) Precordial ST-Segment Elevation in Acute Occlusion of the Proximal Right Coronary Artery. Journal of Electrocardiology, 39, 301-304. https://doi.org/10.1016/j.jelectrocard.2006.02.003

[11] Collins, N., Elliott, V. and Seidelin, P. (2007) True Isolated Right Ventricular Infarction with Tombstone Anterior ST Elevation. Heart, 93, 374. https://doi.org/10.1136/hrt.2006.092890

[12] Finn, A.V. and Antman, E.M. (2003) Images in Clinical Medicine. Isolated Right Ventricular Infarction. New England Journal of Medicine, 349, 1636. https://doi.org/10.1056/NEJMicm030315 